\title{
Vasohibin 1 inhibits Adriamycin resistance in osteosarcoma cells via the protein kinase $B$ signaling pathway
}

\author{
WEI HUANG ${ }^{1}$, YANGGUANG REN ${ }^{2}$ and HUI LIU ${ }^{3}$ \\ ${ }^{1}$ Department of Orthopedics, Shanxian Central Hospital of Shandong Province, Heze, Shandong 274300; \\ ${ }^{2}$ Department of Hand and Foot Surgery, First People's Hospital of Jining City, Jining, Shandong 272000; \\ ${ }^{3}$ Clinical Laboratory of Shanxian Central Hospital of Shandong Province, Heze, Shandong 274300, P.R. China
}

Received April 4, 2017; Accepted November 10, 2017

DOI: $10.3892 / \mathrm{ol} .2018 .8074$

\begin{abstract}
Vasohibin (VASH)1 functions as a negative feedback modulator of angiogenesis in vascular endothelial cells. Mesenchymal VASH1 has been demonstrated to be negatively associated with tumor progression, however studies regarding VASH1 in tumor cells and its functions remain limited. The function of VASH1 in osteosarcoma remains unknown. In the present study, it was confirmed that osteosarcoma cells express decreased levels of VASH1 compared with that expressed by human osteoblast cells. 143B cells with decreased VASH1 expression revealed increased Adriamycin (ADR) resistance compared with U-2OS cells with increased VASH1 expression. Subsequent to manipulating VASH1 expression via transfection, results revealed that overexpression of VASH1 in 143B cells inhibited P-glycoprotein (P-gp) expression and ADR resistance significantly; silencing VASH1 in U-2OS cells enhanced P-gp expression and ADR resistance significantly. Research into the molecular mechanism was performed and the results identified that protein kinase B (AKT) and extracellular signal-related kinase signal pathways were both stimulated by VASH1, but only AKT inhibitor LY294002 was identified to efficiently counteract increases in P-gp expression that had been induced by silencing of VASH1 in U-2OS cells. ADR resistance promoted by silencing VASH1 in U-2OS cells was also counteracted by LY294002. In conclusion, the present study confirmed the low expression of VASH1 in osteosarcoma cells. It was identified that VASH1 was able to inhibit drug resistance in osteosarcoma cells through regulation of P-gp via the AKT signal pathway. This demonstrated a negative regulation function of VASH1 in osteosarcoma, deepened understanding of the function of VASH1 in tumors and suggests a basis for further studies in to the functions of VASH1.
\end{abstract}

Correspondence to: Mrs. Hui Liu, Clinical Laboratory of Shanxian Central Hospital of Shandong Province, 1 Wenhua Road, Heze, Shandong 274300, P.R. China

E-mail: liuhui-0530@163.com

Key words: vasohibin 1, osteosarcoma, Adriamycin resistance, P-glycoprotein, protein kinase B

\section{Introduction}

Osteosarcoma is the most common malignant tumor of the bone and remains the second leading cause of cancer-associated mortality in adolescents globally at present (1). Surgery combined with adjuvant chemotherapy is currently the standard treatment for osteosarcoma (1). In recent years, although a great deal of effort has been made toward improving chemotherapy regimens, the overall prognosis remains poor and much of this may be attributed to drug resistance (2). P-glycoprotein (P-gp), an ATP-binding cassette (ABC) membrane transporter encoded by multidrug resistance 1 (MDR1), is commonly located at the plasma membrane and functions as an ATP-dependent efflux pump for diverse naturally occurring hydrophobic anticancer drugs, including Adriamycin (ADR) (3). Finding an efficient method of inhibiting drug resistance may contribute to better therapeutic outcomes.

Vasohibin (VASH)1 was first identified to be a negative feedback modulator of angiogenesis in vascular endothelial cells in a previous study (4). Inhibitory functions of mesenchymal VASH1 in tumor progression have been reported in different types of tumor (5-7). The functions of parenchymal VASH1 in tumor development have drawn more and more attention, but relevant reports remain limited. Liu et al (2) reported that overexpression of VASH1 in colon cancer cells was able to induce apoptosis and senescence, and inhibited cancer cell growth and colony formation in vitro and tumor growth in vivo. In addition, knockdown of VASH1 in cancer cells was able to promote cell growth, adhesion and migration in vitro and enhance tumorigenesis and metastasis in vivo (8). Takahashi et al (9) reported that VASH1 overexpression in ovarian cells inhibited ovarian cancer growth and peritoneal dissemination and prolonged host survival. Thus far, there remains no report on the functions of VASH1 in osteosarcoma to the best of our knowledge.

In the present study, it was identified that VASH1 is underexpressed in osteosarcoma cells. It was also revealed that VASH1 was able to inhibit ADR resistance of osteosarcoma cells through regulation of the protein kinase B (AKT) signaling pathway. This suggested that further evaluation of VASH1 may yield a novel therapeutic approach to the treatment of osteosarcoma. 


\section{Materials and methods}

Cell culture. The human osteoblast cell line hFOB1.19 and human osteosarcoma cell lines U-2OS and 143B were purchased from American Type Culture Collection (Manassas, VA, USA). All cells were cultured in Dulbecco's modified Eagle's medium (DMEM) (Invitrogen; Thermo Fisher Scientific, Inc., Waltham, MA, USA) supplemented with 10\% fetal bovine serum (FBS; Invitrogen; Thermo Fisher Scientific, Inc.), at $37^{\circ} \mathrm{C}$ in $5 \% \mathrm{CO}_{2}$.

Drug resistance assay. U-2OS and 143B cells were counted and plated in 96-well plate at 10,000 cells/well. After $24 \mathrm{~h}$, the culture medium was replaced with DMEM containing different concentrations $(2,4,8,16,32 \mu \mathrm{mol} / \mathrm{l})$ of ADR (HarveyBio, Inc., Beijing, China). These cells served as experimental groups. Cells in medium without ADR served as the control group. After $48 \mathrm{~h}$, an MTT assay kit (Beijing Solarbio Science \& Technology Co., Ltd., Beijing, China) was used and the optical density (OD) value was measured at $490 \mathrm{~nm}$ wavelength using an ultraviolet spectrophotometer (Shanghai Spectrum Instrument Co., Ltd., Shanghai, China) according to the manufacturer's protocol according to the manufacturer's protocol. Inhibition rate (IR) was calculated using the following equation: IR = 1 - OD value of experiment group/OD value of control group $\mathrm{x} 100 \%$. Half maximal inhibitory concentration $\left(\mathrm{IC}_{50}\right)$ was calculated using regression analysis by SPSS 11.0 software (SPSS, Inc., Chicago, IL, USA). All experiments were repeated 3 times.

Cell transfection. U-2OS and 143B cells were counted and plated in 6 -well plates at $2 \times 10^{5}$ cells/well. After 24 h, p-GPU6/Neo/VASH1 (Shanghai GenePharma Co. Ltd., Shanghai, China) to silence VASH1 expression, and pEZM61/VASH1 (Gene Copoeia, Guangzhou, China) to overexpress VASH1 were transfected using Lipofectamine ${ }^{\circledR} 2000$ (Invitrogen; Thermo Fisher Scientific, Inc.). Empty plasmids were used as control. Reverse transcription-polymerase chain reaction (RT-PCR) and western blotting were performed to confirm transfection efficiency.

$R T-P C R$. RNA was extracted from cells using TRIzol (Life Sciences; Thermo Fisher Scientific, Inc.) according to the manufacturers protocol. cDNA was synthesized using a PrimeScript RT-PCR kit (Takara Biotechnology Co., Ltd., Dalian, China). PCR was performed using specific primers and Universal PCR Master Mix (Thermo Fisher Scientific, Inc.). The thermocycling conditions were as follows; VASH1, $4^{\circ} \mathrm{C}$ for $5 \mathrm{~min}, 94^{\circ} \mathrm{C}$ for $30 \mathrm{sec}, 57^{\circ} \mathrm{C}$ for $30 \mathrm{sec}, 72^{\circ} \mathrm{C}$ for $30 \mathrm{sec}$ for 40 cycles and $72^{\circ} \mathrm{C}$ for $5 \mathrm{~min}$; P-gp, $4^{\circ} \mathrm{C}$ for $5 \mathrm{~min}, 94^{\circ} \mathrm{C}$ for $30 \mathrm{sec}, 54^{\circ} \mathrm{C}$ for $30 \mathrm{sec}, 72^{\circ} \mathrm{C}$ for $30 \mathrm{sec}$ for 36 cycles and $72^{\circ} \mathrm{C}$ for $5 \mathrm{~min}$; GAPDH, $4^{\circ} \mathrm{C}$ for $5 \mathrm{~min}, 94^{\circ} \mathrm{C}$ for $30 \mathrm{sec}, 57^{\circ} \mathrm{C}$ for $30 \mathrm{sec}, 72^{\circ} \mathrm{C}$ for $30 \mathrm{sec}$ for 36 cycles and $72^{\circ} \mathrm{C}$ for $5 \mathrm{~min}$. PCR products were electrophoretically separated on $1.0 \%$ agarose gel. Results were analyzed using Labwork software (version 4; UVP, Inc., Upland, CA, USA). All primers were as follows: VASH1 forward, 5'-CCACGCCCTGATTTCTTAAA-3' and reverse, 5'-CCCTGTCAGAGGTCTGCTCT-3'; P-gp forward, 5'-CCCATCATTGCAATAGCAGG-3' and reverse, 5'-GTT CAAACTTCTGCTCCTGA-3'; GAPDH forward, 5'-AGA
AGGCTGGGGCTCATTTG-3' and reverse, 5'-AGGGGCCAT CCACAGTCTTC-3' GAPDH served as an internal control. All experiments were repeated 3 times.

Western blotting. Protein was extracted from cells using radioimmunoprecipitation assay lysis containing $1 \%$ phenylmethane sulfonyl fluoride (Beyotime Institute of Biotechnology, Haimen, China) and protein concentration was analyzed using a bicinchoninic acid assay kit (Pierce; Thermo Fisher Scientific, Inc.). Equal quantities of protein were loaded per well in 5\% acrylamide and separated using 10\% SDS-PAGE, and transferred to nitrocellulose membrane. The membrane was incubated with primary antibodies (Table I) at $4^{\circ} \mathrm{C}$ overnight, and then in horseradish peroxidase-conjugated goat anti-rabbit secondary antibody (cat. no. ab205718; 1:4,000; Abcam, Cambridge, MA, USA) at room temperature for $1 \mathrm{~h}$. Signals were detected using enhanced chemiluminescence reagents (Pierce; Thermo Fisher Scientific, Inc.) and quantified using Image-Pro software (version 5.1; Media Cybernetics, Inc., Rockville, MA, USA). All experiments were repeated 3 times.

Statistical analysis. SPSS software (version 11.0; SPSS, Inc., Chicago, IL, USA) was used and data were expressed as mean \pm standard deviation. Differences between groups were analyzed using one-way analysis of variance with Dunnett's post hoc test. $\mathrm{IC}_{50}$ was calculated using regression analysis. $\mathrm{P}<0.05$ was considered to indicate a statistically significant difference.

\section{Results}

VASH1 is expressed weakly in osteosarcoma cells. VASH1 expression was exhibited both at RNA (Fig. 1A) and protein (Fig. 1B) levels. Compared with human osteoblast cell lines hFOB1.19, decreased VASH1 expression was detected in osteosarcoma cell lines U-2OS and 143B. VASH1 expression was significantly decreased in 143B cells compared with that in $\mathrm{U}-2 \mathrm{OS}$ cells. A drug resistance assay was subsequently performed, revealing that the inhibition rate (IR) of 143B cells in ADR was decreased compared with that of U-2OS (Fig. 1C). The $\mathrm{IC}_{50}$ of $143 \mathrm{~B}$ cells $(6.59 \pm 0.89 \mu \mathrm{mol} / \mathrm{l})$ was significantly increased compared with that of U-2OS cells (4.32 $\pm 0.47 \mu \mathrm{mol} / 1$; Fig. 1D). All these results indicate possible associations between VASH1 expression and drug resistance.

VASH1 inhibits the ADR resistance of osteosarcoma cells. To confirm whether VASH1 was able to regulate drug resistance of osteosarcoma cells, VASH1 expression was manipulated through transfection. Following overexpression of VASH1 in 143B cells, P-glycoprotein (P-gp) expression was significantly inhibited at both the RNA (Fig. 2A) and protein (Fig. 2B) levels . The IR of 143B cells was increased compared with control cells (Fig. 2C). $\mathrm{IC}_{50}$ declined from $7.14 \pm 0.83$ to $3.79 \pm 0.56 \mu \mathrm{mol} / 1$ (Fig. 2D). Following silencing of VASH1 in U-2OS cells, P-gp expression was upregulated both at RNA (Fig. 2E) and protein (Fig. 2F) levels. IR of U-2OS cells declined significantly (Fig. $2 \mathrm{G}$ ), $\mathrm{IC}_{50}$ increased from $4.32 \pm 0.88$ to $7.34 \pm 0.69$ or $6.71 \pm 0.82 \mu \mathrm{mol} / 1$ (Fig. $2 \mathrm{H}$ ). All results suggested the inhibitory function of VASH1 in ADR resistance. 
Table I. Primary antibodies used in western blotting.

\begin{tabular}{llll}
\hline Name & Cat. no. & Dilution & Supplier \\
\hline VASH1 & Ab199732 & $1: 300$ & Abcam, Cambridge, UK \\
P-gp & Ab170904 & $1: 400$ & Abcam, Cambridge, UK \\
phospho-ERK1/2 & AF1018 & $1: 1,000$ & Cell Signaling Technology, Inc., Danvers, MA, USA \\
ERK1/2 & AF1576 & $1: 1,000$ & Cell Signaling Technology, Inc., Danvers, MA, USA \\
phospho-AKT & AF887 & $1: 1,000$ & Cell Signaling Technology, Inc., Danvers, MA, USA \\
AKT & AF2055 & $1: 1,000$ & Cell Signaling Technology, Inc., Danvers, MA, USA \\
GAPDH & Ab9485 & $1: 4,000$ & Abcam, Cambridge, UK
\end{tabular}

VASH, Vasohibin; P-gp, p-glycoprotein; phospho, phosphorylated; ERK, extracellular signal-related kinase; AKT, protein kinase B.

A

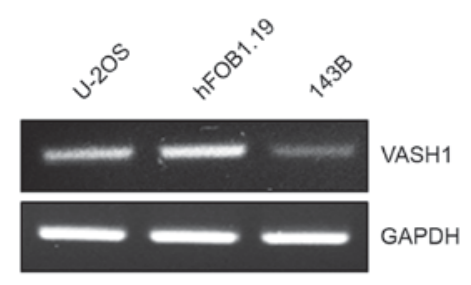

$\mathrm{B}$
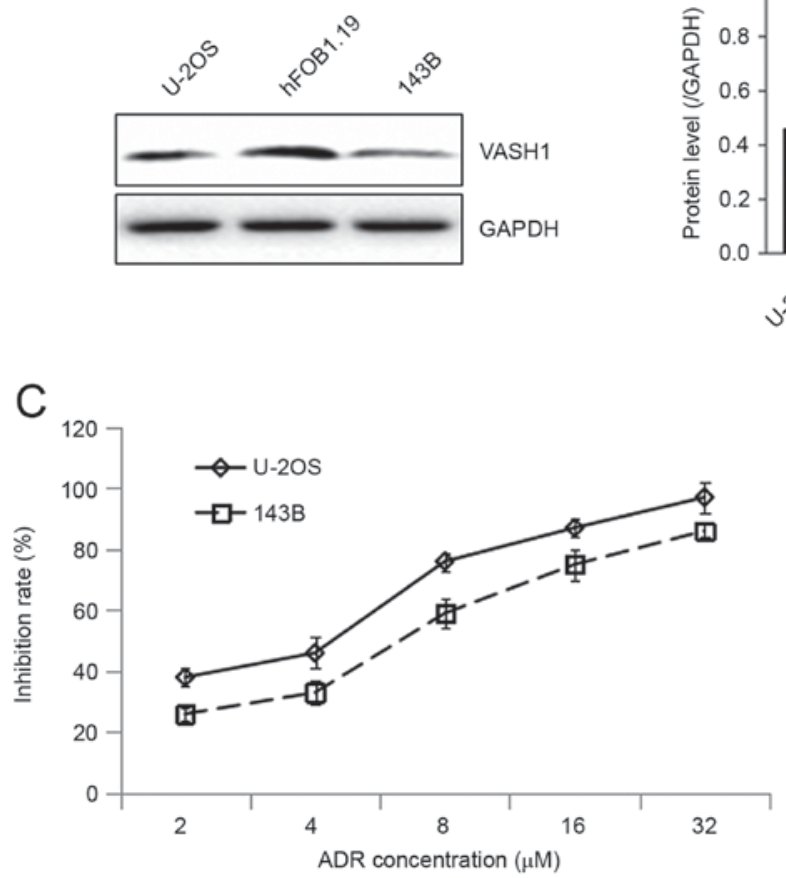
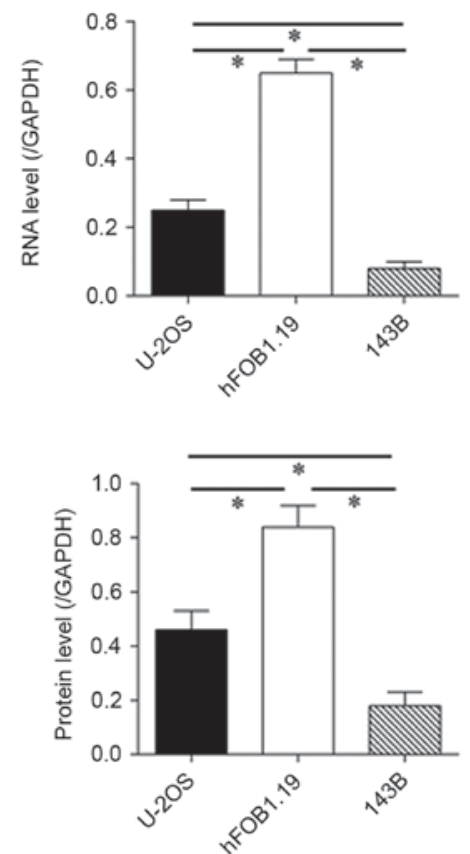

D

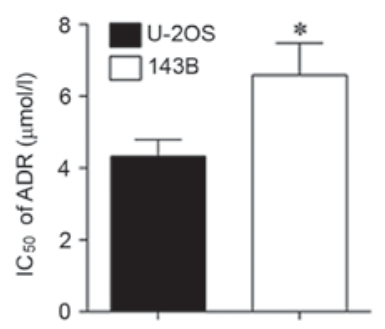

Figure 1. VASH1 expression status in osteosarcoma cells and ADR resistance. VASH1 expression in the human osteoblast cell line hFOB1.19 and the human osteosarcoma cell lines $\mathrm{U}-2 \mathrm{OS}$ and $143 \mathrm{~B}$ was detected using (A) reverse transcription-polymerase chain reaction and (B) western blot analysis. ${ }^{*} \mathrm{P}<0.05$ (hFOB1.19 vs. U-2OS and 143B). (C) ADR resistance of U-2OS and 143B cells was detected using a drug resistance assay. (D) IC50 of U-2OS and 143 cells in ADR. ${ }^{*} \mathrm{P}<0.05$ (U-2OS vs. 143B). ADR, Adriamycin; VASH, Vasohibin.

VASH1 regulation of ADR resistance uses the AKT signaling pathway. As presented in Fig. 3A, following overexpression of VASH1 in 143B cells, phosphorylation of extracellular signal-related kinase (ERK) and AKT was inhibited (Fig. 3A). Conversely, following silencing of VASH1 in U-2OS cells, phosphorylation of ERK and AKT was upregulated (Fig. 3B). Once AKT inhibitor LY294002 was added, the increase of P-gp in U-2OS cells induced by silencing VASH1 was decreased (Fig. 3C). However, with ERK inhibitor U0126 added, no change was observed in P-gp expression (Fig. 3D). 
A

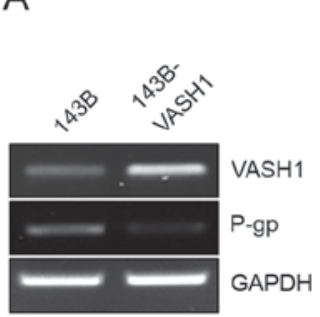

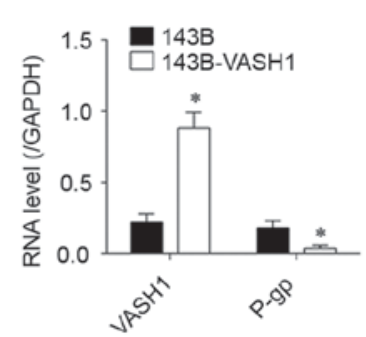

C

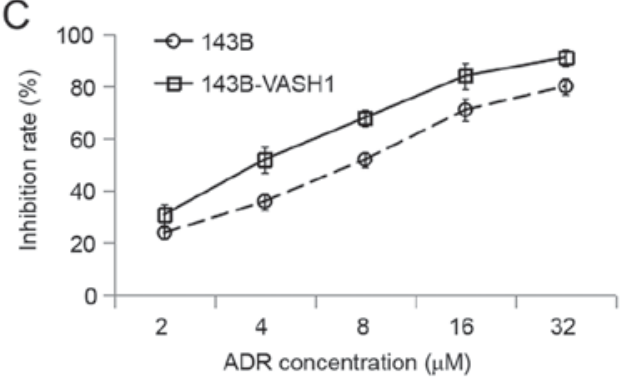

B

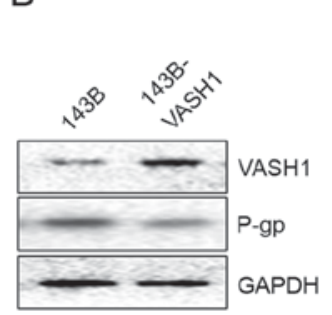

D

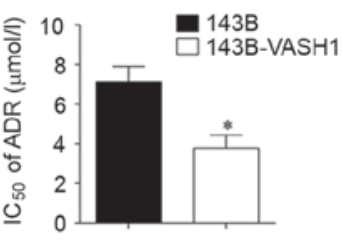

E
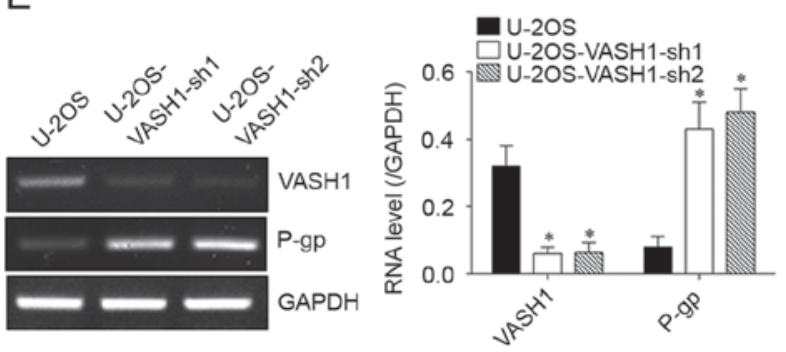

$\mathrm{F}$
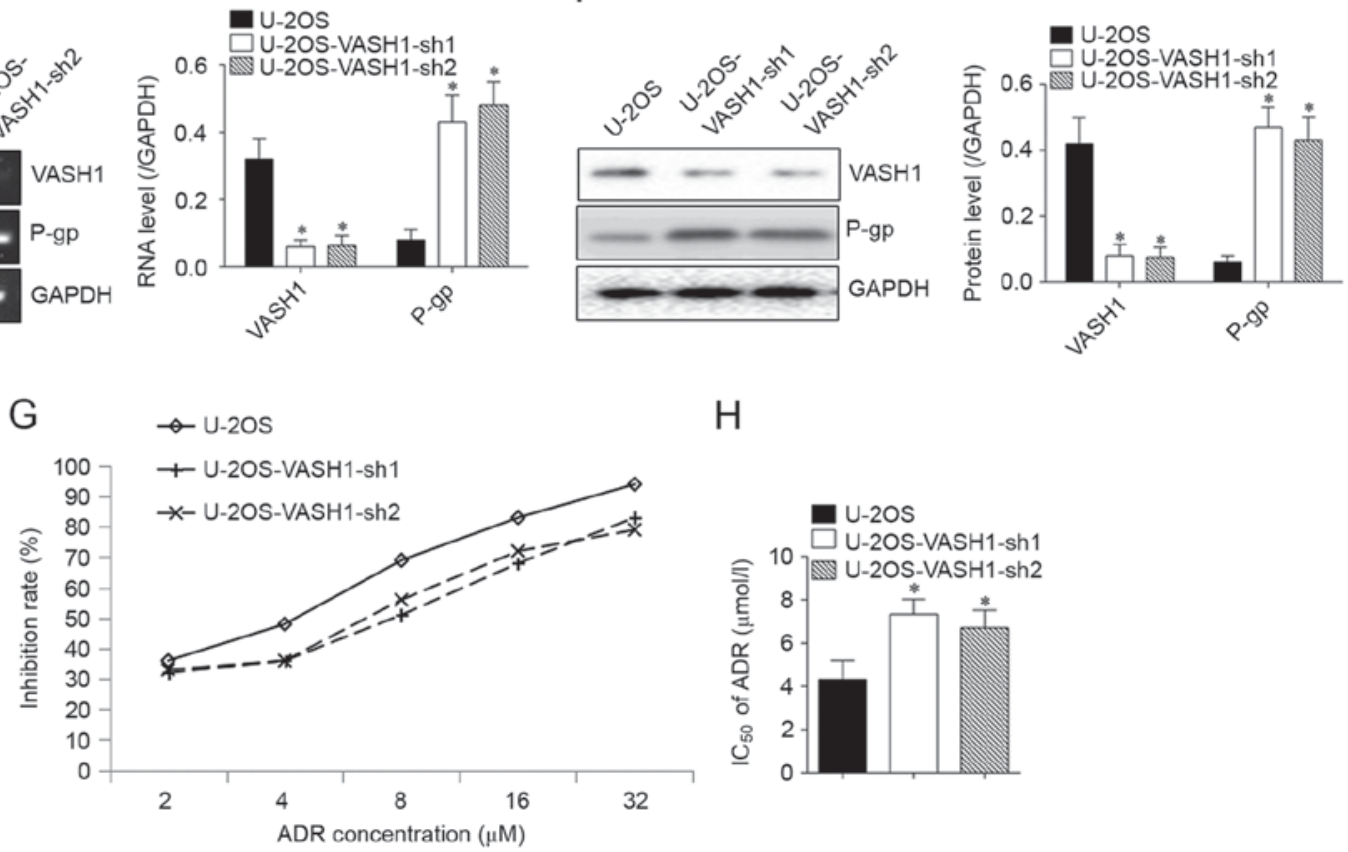

$\mathrm{H}$

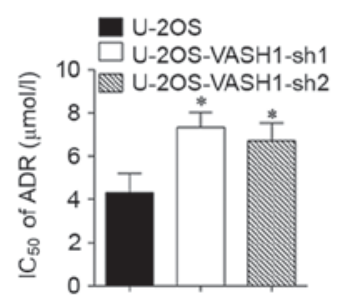

Figure 2. VASH1 inhibits ADR resistance of osteosarcoma cells. Following overexpression of VASH1 in 143B cells, P-gp expression declined at both the (A) RNA and (B) protein levels. (C) The inhibition rate of $143 \mathrm{~B}$ cells in ADR increased and (D) $\mathrm{IC}_{50}$ declined significantly ${ }^{*} \mathrm{P}<0.05$ vs. $143 \mathrm{~B}$ cells. Following silencing of VASH1 in U-2OS cells, P-gp expression increased at both the (E) RNA and (F) protein levels. (G) The inhibition rate of U-2OS cells in ADR declined with VASH1 silenced and (H) $\mathrm{IC}_{50}$ increased significantly. ${ }^{*} \mathrm{P}<0.05$ vs. U-2OS cells. ADR, Adriamycin; VASH, Vasohibin; P-gp, p-glycoprotein.

A drug resistance assay also revealed that LY294002 could counteract the decrease of IR of U-2OS cells in ADR induced by silencing VASH1, but U0126 did not influence declination of IR of U-2OS cells in ADR induced by silencing VASH1. This suggests that the AKT signaling pathway may serve a function in ADR resistance regulated by VASH1 (Fig. 3E).

\section{Discussion}

A member of the vasohibin family, the human VASH1 gene is located on chromosome 14q24.3. VASH1 protein is composed of 365 amino acids with no glycosylation sites $(10,11)$. Vasohibin 2 is also a member of the vasohibin family and was initially known as an angiogenic factor. VASH1 was first noticed for its ability to inhibit angiogenesis; it is restricted in vessel endothelial cells and several other types of cell (12). The negative regulation of VASH1 from tumor cells on tumor progression has been demonstrated in colon cancer (8), ovarian (9) and renal carcinoma (13). However, in 2014, Kitajima et al (14) reported that high VASH1 in the cytoplasm of colorectal cancer (CRC) tissues was positively associated with tumor progression, and silencing VASH1 inhibited CRC cell proliferation, migration and invasion, and promoted anoikis. Thus, the functions of VASH1 in different types of tumor are not consistent, therefore the effects of VASH1 on osteosarcoma require further investigation. 
A

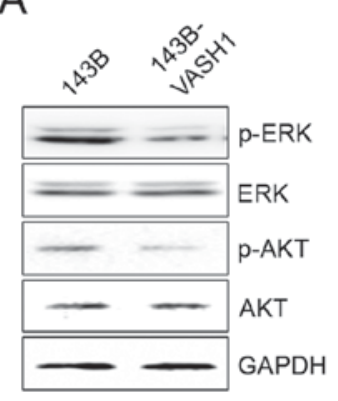

D

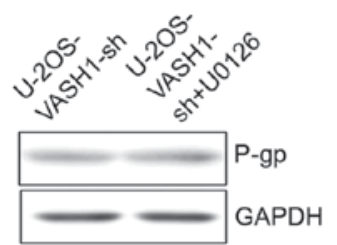

B

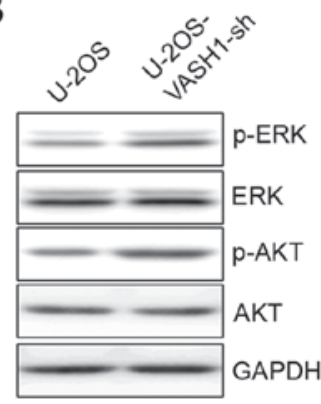

C
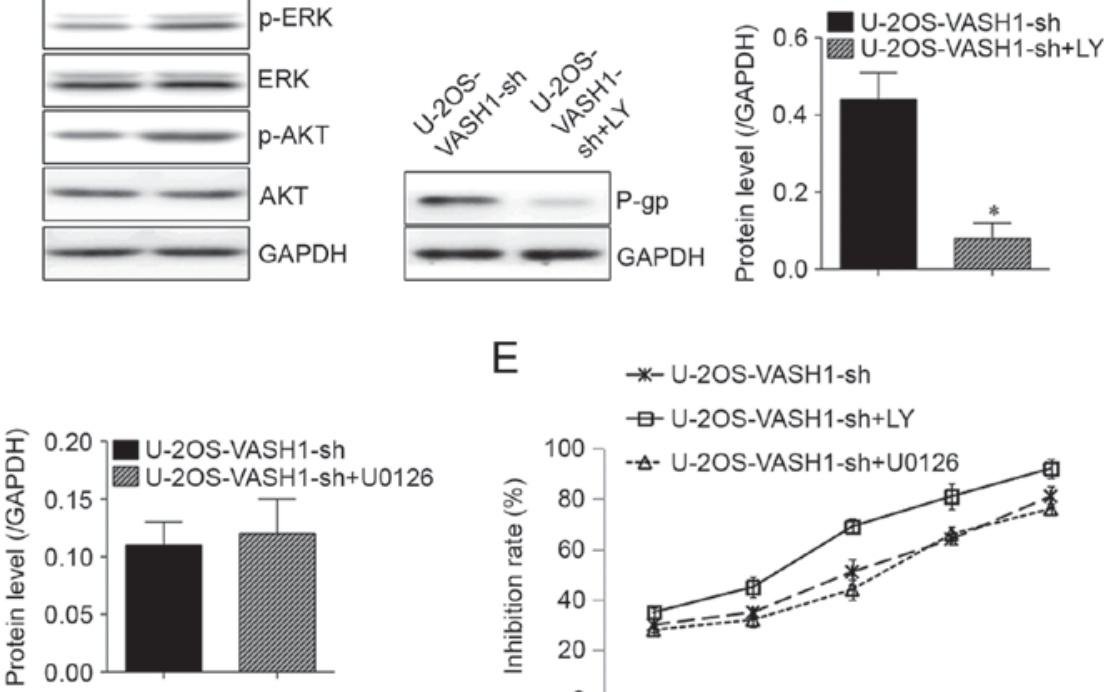

E

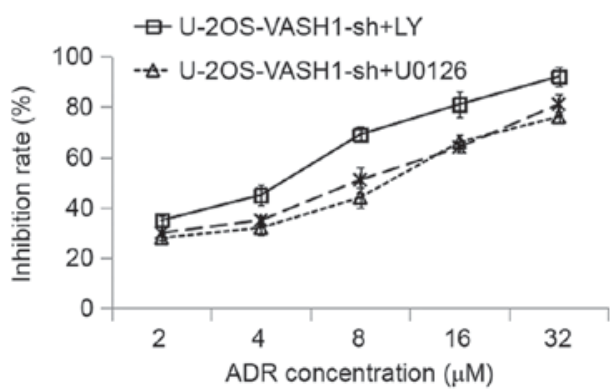

Figure 3. ADR resistance regulated by VASH1 relies on the AKT signal pathway. (A) Subsequent to overexpressing VASH1 in 143B cells, phosphorylation of AKT and ERK declined. (B) Subsequent to silencing of VASH1 in U-2OS cells, phosphorylation of AKT and ERK increased. (C) AKT inhibitor LY294002 inhibited P-gp expression and (E) ADR resistance in U-2OS cells induced by silencing VASH1 ${ }^{*} \mathrm{P}<0.05$ vs. U-2OS cells with silenced VASH1. ERK inhibitor U0126 exhibited no effect on (D) P-gp expression or (E) ADR resistance in U-2OS cells induced by silencing VASH1. ADR, Adriamycin; VASH, Vasohibin; P-gp, p-glycoprotein; AKT, protein kinase B; ERK, extracellular signal-related kinase.

Drug resistance is an important characteristic of malignant tumors and has been an important factor in the failure of cancer treatment (15). ATP-binding cassette drug efflux pump P-gp has been proposed to serve crucial functions for tumor cells acquiring MDR $(16,17)$. ADR is the first-line chemotherapy drug used to treat osteosarcoma. It not only inhibits DNA transcription and replication but also induces breakage of DNA double strands (18). In the present study, data revealed low expression of VASH1 in osteosarcoma cells at both the RNA and protein levels. Furthermore, osteosarcoma cells with lower VASH1 levels exhibited more marked ADR resistance. This suggests that VASH1 may serve negative regulatory functions in osteosarcoma drug resistance. Through changing VASH1 using transfection, it was identified that VASH1 was able to inhibit the P-gp expression and ADR resistance of osteosarcoma cells. This is consistent with the negative regulatory functions of VASH1 reported by the majority of works $(8,9,13)$, but inconsistent with a report from Kitajima et al (14). Different organs of origin of different tumors may explain this divergence.

The AKT and ERK signaling pathways may be stimulated in different types of tumors. This activates proliferation and survival signals that ultimately lead to tumorigenesis and progression (19). In 2016, Yang et al (20) reported that the ERK signal pathway may serve important functions in 5-FU-mediating of colorectal cancer. Xiao et al (21) identified that Oridonin inhibits gefitinib-resistant lung cancer cells by suppressing ERK and AKT signaling pathways. In the present study, it was identified that VASH1 downregulated P-gp expression by blocking the AKT signal pathway, thus inhibiting ADR resistance of osteosarcoma cells. In the present study, no effects of ERK were observed in ADR resistance. This is not consistent with relevant reports from Yang et al (20) and Xiao et al (21). This may be attributed to differences in the type of tumor and drugs with different anti-tumor mechanisms.

To conclude, the inhibitory effects of VASH1 on osteosarcoma drug resistance were confirmed. This has enhanced understanding of the functions of VASH1 in tumors and supplied a basis for ongoing studies targeting VASH1. VASH1 may be treated as an enhancer of chemotherapeutic sensitivity in osteosarcoma cells to foster better prognosis.

\section{Acknowledgements}

Not applicable.

\section{Funding}

No funding was received.

\section{Availability of data and materials}

All data generated or analyzed during this study are included in this published article.

\section{Authors' contributions}

HL designed this study. WH performed the experiments. YR analyed the results.

\section{Ethics approval and consent to participate}

Not applicable. 


\section{Consent for publication}

Not applicable.

\section{Competing interests}

The authors declare that they have no competing interests.

\section{References}

1. Sobhan MR, Forat Yazdi M, Mazaheri M, Zare Shehneh M and Neamatzadeh H: Association between the DNA repair gene XRCC3 rs861539 polymorphism and risk of osteosarcoma: A systematic review and meta-analysis. Asian Pac J Cancer Prev 18: 549-555, 2017.

2. Liu R, Fu C, Sun J, Wang X, Geng S, Wang X, Zou J, Bi Z and Yang C: A new perspective for osteosarcoma therapy: Proteasome inhibition by MLN9708/2238 successfully induces apoptosis and cell cycle arrest and attenuates the invasion ability of osteosarcoma cells in vitro. Cell Physiol Biochem 41: 451-465, 2017.

3. Colabufo NA, Contino M, Cantore M, Capparelli E, Perrone MG, Cassano G, Gasparre G, Leopoldo M, Berardi F and Perrone R Naphthalenyl derivatives hitting for P-gp/MRP1/BCRP transporters. Bioorg Med Chem 21: 1324-1332, 2013.

4. Coch L, Mejias M, Berzigotti A, Garcia-Pras E, Gallego J, Bosch J, Mendez R and Fernandez M: Disruption of negative feedback loop between vasohibin-1 and vascular endothelial growth factor decreases portal pressure, angiogenesis, and fibrosis in cirrhotic rats. Hepatology 60: 633-647, 2014.

5. Watanabe K, Hasegawa Y, Yamashita H, Shimizu K, Ding Y, Abe M, Ohta H, Imagawa K, Hojo K, Maki H, et al: Vasohibin as an endothelium-derived negative feedback regulator of angiogenesis. J Clin Invest 114: 898-907, 2004.

6. Miyashita H, Watanabe T, Hayashi H, Suzuki Y, Nakamura T, Ito S, Ono M, Hoshikawa Y, Okada Y, Kondo T and Sato Y: Angiogenesis inhibitor vasohibin-1 enhances stress resistance of endothelial cells via induction of SOD2 and SIRT1. PLoS One 7: e46459, 2012.

7. Miyashita H, Suzuki H, Ohkuchi A and Sato Y: Mutual balance between vasohibin-1 and soluble VEGFR-1 in endothelial cells. Pharmaceuticals 4: 782-793, 2011.

8. Liu S, Han B, Zhang Q, Dou J, Wang F, Lin W, Sun Y and Peng G: Vasohibin-1 suppresses colon cancer. Oncotarget 6: 7880-7898, 2015.

9. Takahashi Y, Saga Y, Koyanagi T, Takei Y, Machida S, Taneichi A, Mizukami H, Sato Y, Matsubara S and Fujiwara H: The angiogenesis regulator vasohibin-1 inhibits ovarian cancer growth and peritoneal dissemination and prolongs host survival. Int J Oncol 47: 2057-2063, 2015.
10. Zhang T, Yu TT, Zhang DM, Hou XM, Liu XJ, Zhao D and Shan L: Vasohibin-1 expression detected by immunohistochemistry correlates with prognosis in non-small cell lung cancer. Med Oncol 31: 963, 2014.

11. Yan Y, Shen Z, Ye Y, Jiang K, Zhang H, Shen C, Mustonen H, Puolakkainen P and Wang S: A novel molecular marker of prognosis in colorectal cancer: Vasohibin-1. Med Oncol 31: 816, 2014.

12. Kern J, Steurer M, Gastl G, Gunsilius E and Untergasser G: Vasohibin inhibits angiogenic sprouting in vitro and supports vascular maturation processes in vivo. BMC Cancer 9: 284, 2009.

13. Zhao G, Yang Y, Tang Y, Han R and Sun Y: Reduced expression of vasohibin-1 is associated with clinicopathological features in renal cell carcinoma. Med Oncol 29: 3325-3334, 2012.

14. Kitajima T, Toiyama Y, Tanaka K, Saigusa S, Kobayashi M, Inoue Y, Mohri Y and Kusunoki M: Vasohibin-1 increases the malignant potential of colorectal cancer and is a biomarker of poor prognosis. Anticancer Res 34: 5321-5329, 2014.

15. He S, Shen J, Hu N, Xu X and Li J: DKK4 enhances resistance to chemotherapeutics 5-Fu and YN968D1 in colorectal cancer cells. Oncol Lett 13: 587-592, 2017.

16. Tandia M, Mhiri A, Paule B, Saffroy R, Cailliez V, Noé G, Farinotti R and Bonhomme-Faivre L: Correlation between clinical response to sorafenib in hepatocellular carcinoma treatment and polymorphisms of P-glycoprotein (ABCB1) and of breast cancer resistance protein (ABCG2): Monocentric study. Cancer Chemother Pharmacol 79: 759-766, 2017.

17. Esser L, Zhou F, Pluchino KM, Shiloach J, Ma J, Tang WK, Gutierrez C, Zhang A, Shukla S, Madigan JP, et al: Structures of the multidrug transporter P-glycoprotein reveal asymmetric ATP binding and the mechanism of polyspecificity. J Biol Chem 292: 446-461, 2017.

18. Zhao M, Yu S and Zhang M: Differential expression of multidrug resistance-related proteins in Adriamycin-resistant (pumc-91/ ADM) and parental (pumc-91) human bladder cancer cell lines. Mol Med Rep 14: 4741-4746, 2016

19. Wu YL, Maachani UB, Schweitzer M, Singh R, Wang M, Chang R and Souweidane MM: Dual inhibition of PI3K/AKT and MEK/ERK pathways induces synergistic antitumor effects in diffuse intrinsic pontine glioma cells. Transl Oncol 10: 221-228, 2017.

20. Yang K, Gao K, Hu G, Wen Y, Lin C and Li X: CTGF enhances resistance to 5-FU-mediating cell apoptosis through FAK/MEK/ ERK signal pathway in colorectal cancer. Onco Targets Ther 9: 7285-7295, 2016

21. Xiao X, He Z, Cao W, Cai F, Zhang L, Huang Q, Fan C, Duan C, Wang $\mathrm{X}$, Wang $\mathrm{J}$ and Liu Y: Oridonin inhibits gefitinib-resistant lung cancer cells by suppressing EGFR/ERK/MMP-12 and CIP2A/Akt signaling pathways. Int J Oncol 48: 2608-2618, 2016. 